\title{
NOTE DE L'EDITEUR :-
}

"Notre Seigneur Jésus Christ a dit quelque part: 'Que ceux qui peuvent comprendre comprennent.

(J. ROMAINS, Les Copains.)

C'est encore à notre correspondant permanent auprès des Archives de la Voirie Municipale de Tegucigalpa (Guatemala) que nous devons le document historique que nous publions ici. $S i$ l'on peut douter sérieusement du caractère original du susdit document, son caractere apocryphe est au-dessus de tout soupçon. Nous tenions. par souci d'honnêteté vis-á-vis de nos lecteurs, de nous-mêmes, de la science, de la Postérité et.du Syndicat des Agents de Voirie Tegucigalpiens, à éclaircir ce point avant de soumettre a l'appréciation des foules l'un des plus grands chefs-d'oeuvres parahistoricolittéraires de tous les temos: La Geste Toto.

Les divers manuscrits de la Geste Toto sont conservés respectivement à Tegucigalpa, Dudweiler, Postdam, Ur, Smolensk, Marcq-en-Baroeul, Socomme, Gomorrhe, Carpentras, ainsi qu'en dix-neuf autres lieux, sans intérêt aucun et dont la liste ne pourrait ici qu'importuner le lecteur, I'indisposer à notre égard, et--qui sait--l'amener à contracter d'étranges maladies toutes plus graves les unes que les autres et auxquelles il succomberait rapidement, ajoutant ainsi son nom au bas d'une autre liste--celle de's victimes innocentes de la Science--liste que, pour les raisons nombreuses et, $n$ 'hésitons pas à le dire, fort pertinentes que nous venons de mentionner, nous nous abstiendrons de publier. (Le lecteur dont la santé ne serait pas a toute epreuve peut, s'il en exprime le désir , se dispenser de lire ce paragraphe.)

Tresvait la nuit ed apert l'albe clere,

Li ber Toto est repaidriez de feste;

De son pedre la charete at fraite:

Ia riedre-guarde tant par est peceide

Desclóse est la porte, desmalét li costez 
E altresi sont les eles estrossedes.

Ja vostre charz nen ait meisme soffraite

5745

Ço dist Toto: "Si mare fut neț:

Com pesmes jorz m'est hui ajornez!"

En son visage est molt descolorez;

Baisset son chief, comencet a penser

Pesmes est son ped̆re, at la main legiere.

5750

Las est $1 i$ ber, si s'est colchiez a tere;

Toto se dort que mie ne s'esveillet. Aoi.

Toto se dort que ne s'esveillet mie

Ii soleilz fut clers e halz e chalz e sonet at midi, midi è demi.

or vint $i_{i}$ quens e vist son fils gesir.

5755

Il est merveillos hom plus que jo ne sai dire;

Mien escient at $1 i$ quens grant ire.

Si overte af la boche ed a braire se prist:

"Ahi! Culverz, malvais hom, barbarin,

Sathanas, gloton, e felon encrisme!"

5760

Lors s'esveillet Toto e veit son pedre e dist:

"Tresvait la nuit ed apert l'albe clere,

Ii ber Toto est repádoriez de feste'

De son pedre...

NOTES

5739. V. donne: "Tresvait la nuit du 23 au 27 septembre $1128 . . . "$ " ce qui permet de situer cette oeuvre dans son contexte historique avec toute $I^{\prime}$ exactitude désirable en pareille matière.

5740. V4 donne: "Ii ber Iéon Trotsky; "I'emploi de I'article défini est ici grammaticalement justifié, et n'appelle aucune remarque. V7, notons-le cependant, donne: "La ber Toto," mais il s'agit lá d'un pâté. Sur l'interprétation des pâtés dans la Geste Toto, voir RHLG, XXVI (1913), p. 13-904 (H. von Grossbliederstroff).

5741. $\mathrm{V}^{4}$ donne: "De sxn fredre." $\mathrm{v}^{7}$ donne: "De son oncle." $\mathrm{V}^{8}$ : "De son concessionedre." Toto avait I'habitudecomme cela se pratiquait couramment à l'époque-- 
d'emprunter le véhicule de ses parents, amis ou relations. Cette coutume a survécu jusqu'á nos jours. Sur ce point voir C.A.P.E.亡.E.E.P.S.S. (1937-1967). 5744. V2 donne: "les eles knrvzhtühbmjtw. "C'est douteux. 5745. A rapprocher de Rol. V. 2257.

5749. La conduite de Toto peut sembler singulière; elle est cependant facile à comprendre: c'est tout ce qu'il peut faire, a moins qu'il ne quitte le domicile paternel. Sur cette dernière possibilité, voir "La vie de Saint Alexis."

5750. C. 5741.

5751. $\mathrm{v}^{5}$ donne "Rodon es lo ber," cê dont on peut déduire d'une part que le scribe de $\mathrm{V}^{3}$ manquait de bases solides tant en grammaire qu'en orthographe et d'autre part que le baron Toto se serait ignominieusement saoulé au cours de la fête mentionnée au vers 5740 . Cette hypothese est confirmée par tous les manuscrits que nous possédons, remarquons-le au passage. Si nous avons cru bon de substituer "las," c'est dans le souci de rendre Toto un peu moins odieux qu'il n'est dans les autres versions. Nous avons cru que l'ivresse avait quelque chose de trop bas et de trop noir pour que notre héros pût s'y, abandonner pendant la semaine (cf. 5739.), en début de semaine qui plus est.

5753. A rapprocher de 5752.

5754. Vers douteux pour le rythme; n'existe dans aucune des versions.de la Geste Toto. Purement et simplement rajouté par nos soins.

5757. On le serait à moins.

5758. Observation dont la précision n'a d'égal que le réalisme.

5760. On dit habituellement encrisme felon.

5762. Voir 5739 et suivants. Il s'agit d'une série interminable de laisses exactement semblables, similaires et identiques. 


\section{TABLE DES ABBREVIATIONS}

-CAPELEEPSSA. Cahiers de l'Association des Parents d'Elèves des Etablissements d'Enseignement Primaire, secondaire, Supérieur et Autres.

-RHLG. Revue d'Histoire Iittéraire du. Guatenala.

-Rol. T,a Chanson de Roland, Oxford version; edtuion, notes anc: glossary by T. AtJinson JENKINS, D. C. Heath and Company pub. Boston, 1965.

-Sch. W. SCHULTZ, Das altfranzösiche Totoslied nach der Tegucigalpaner Handschrift. Dudweiler, 1913.

- V. La geste Toto, manuscrit de Tegucigalpa. $-V^{1}$ à $V^{27}$. Différents manuscrits, déposés en divers lieux. $-\mathrm{v}^{27} \mathrm{a} \mathrm{v}^{52}$. (Pour mémoire) Manuscrits itinérants. 